\title{
Restoration of the Spinous Process Following Muscle-Preserving Posterior Lumbar Decompression via Sagittal Splitting of the Spinous Process
}

\author{
Seung Myung Wi, MD, Hui Jong Lee, MD, Sam Yeol Chang, MD, Oh Hyo Kwon, MD, \\ Choon-Ki Lee, MD, Bong-Soon Chang, MD, Hyoungmin Kim, MD
}

Department of Orthopedic Surgery, Seoul National University Hospital, Seoul National University College of Medicine, Seoul, Korea

Background: In lumbar spinal stenosis, spinous process-splitting decompression has demonstrated good clinical outcomes with preservation of the posterior ligamentous complex and paraspinal muscles in comparison to conventional laminectomy, but the radiological consequence and clinical impact of the split spinous processes have not been fully understood.

Methods: Seventy-three patients who underwent spinous process-splitting decompression were included. The bone union rate and pattern were evaluated by computed tomography performed 6-18 months after surgery and compared among subgroups divided according to the number of levels decompressed and the extent of spinous process splitting. The bone union pattern was classified into three categories: complete union, partial union, and nonunion. The visual analog scale (VAS) score, Oswestry disability index (ODI), and walking distance assessed both before and 24-36 months after surgery were compared among subgroups divided according to the union pattern of the split spinous process.

Results: Overall, the rates of complete union, partial union, and nonunion were $51.7 \%, 43.2 \%$, and $5.1 \%$, respectively. In the subgroup with partial splitting of the spinous process, the rates were $85.7 \%, 14.3 \%$, and $0 \%$, respectively; those of the subgroup with total splitting of the spinous process were $32.9 \%, 59.2 \%$, and $7.9 \%$, respectively. With single-level decompression, a higher rate of union was observed compared with multilevel decompression. The VAS, ODI, and walking distance were significantly improved after surgery and did not differ according to the degree of union of the split spinous process.

Conclusions: We found that the single-level operation and partial splitting of the spinous process were favourable factors for obtaining complete restoration of the posterior bony structure of the lumbar spine in spinous process-splitting decompression.

Keywords: Lumbar spinal stenosis, Decompression, Spinous process splitting, Bony union, Clinical outcomes

In lumbar spinal stenosis, the classic laminectomy technique utilises extensive stripping of the bilateral paraspinal muscle from the spinous processes, laminae, and facet

Received September 12, 2018; Accepted December 4, 2018

Correspondence to: Hyoungmin Kim, MD

Department of Orthopedic Surgery, Seoul National University Hospital, Seoul National University College of Medicine, 101 Daehak-ro, Jongnogu, Seoul 03080, Korea

Tel: +82-2-2072-3057, Fax: +82-2-764-2718

E-mail: hmkim21@gmail.com joints and complete removal of the spinous process and laminae in order to decompress the neural tissues. As a result, the paravertebral muscles lose their insertion and become denervated and the posterior ligamentous complex and posterior neural arch are sacrificed, all of which are associated with significant back muscle atrophy, mechanical back pain, spinal segmental instability, and even failed back surgery syndrome. ${ }^{1-9)}$

To overcome these problems, various surgical techniques have been developed. Watanabe et al.'s ${ }^{10,11)}$ technique of laminectomy that utilises the surgical corridor 
formed by sagittal splitting of the spinous process and maintains the attachment of the paravertebral muscle insertion demonstrated good clinical outcomes with reduced postoperative wound pain and minimised injury to the paraspinal muscles. Further modifications of the spinous process-splitting technique by other authors have also been reported to produce satisfactory clinical outcomes, with the advantages of reduced postoperative wound pain, faster recovery, reduced fatty infiltration to the multifidus and other paravertebral muscles, reduced iatrogenic spinal segmental instability following laminectomy and other injuries to the paravertebral muscles during surgery in comparison to the conventional posterior spinal approach using periosteal stripping of the paravertebral muscles. ${ }^{10-20)}$

However, there still exists controversies for the effectiveness of the novel technique. Lee and Lee ${ }^{21)}$ criticised the effectiveness of the novel approach for adequate decompression, particularly in the area of the lateral recess, reporting their initial experience of 25 cases using this novel technique. Rajasekaran et al., ${ }^{22)}$ in their randomised controlled study, deferred judgment on whether the novel spinous-splitting decompression technique was superior to the conventional technique. Furthermore, the fate and benefit of the split spinous process is not yet clearly known. The reported union rate of the spinous process shows wide variations, and there are conflicting opinions on whether it heals in a functional shape that provides a stable attachment site for the paravertebral muscles or merely fails to form a union at the spinolaminar junction, resulting in a floated, unattached, spinous process with loss of the lever-arm function as an attachment site for the paravertebral muscles. ${ }^{10,14,16,21,23)}$ Such nonunion of the split spinous process was even thought to be associated with persistent postoperative pain or the long-term aggravation of back and leg pain. ${ }^{20,21)}$ Within this study, the authors analyzed the union rate and pattern of split spinous processes both between the split spinous process and at the spinolaminar junction, as well as the clinical outcome of patients who underwent decompression for lumbar spinal stenosis via the authors' modified surgical technique based on the spinous process-splitting laminectomy introduced by Watanabe et al. ${ }^{10)}$ and modified by Nomura et al. ${ }^{14)}$

\section{METHODS}

A retrospective analysis of 73 consecutive patients who underwent spinous process-splitting decompression for lumbar spinal stenosis between September 2014 and March 2016 was performed according to the STROBE guideline with approval of Institutional Review Board of Seoul National University Hospital (IRB No. H-1609-094793). Our ethical committee waived the requirement for patients' written informed consent because of the anonymous data collection and the retrospective nature of our study.

\section{Patient Selection and Data Acquisition}

The inclusion criteria of this study were as follows: (1) a radiological finding of lumbar spinal canal stenosis, (2) clinical symptoms and neurological signs corresponding to the level of stenosis on magnetic resonance imaging (MRI), and (3) persistence of symptoms for more than 6 months despite conservative management. The exclusion criteria were as follows: (1) definite instability of the lumbar spine (but grade I spondylolisthesis without significant instability was also included), (2) stenosis due to congenital, spondylolytic, iatrogenic causes, (3) symptoms resulting from peripheral arterial disease, and (4) previous surgery to the lumbar spine.

As part of the radiological assessment, plain radiographs showing standing anteroposterior, lateral, and flexion-extension lateral views acquired before surgery and at 24-36 months after surgery were compared to assess spinal instability. To evaluate bony union both (1) between the split spinous processes and (2) at the spinolaminar junction, computed tomography (CT) images were acquired at 6-18 months after surgery. The union rate and pattern were also compared between subgroups divided according to the number of decompressed levels and the extent of the split spinous process; i.e., total splitting as per Watanabe et al. ${ }^{10)}$ or partial splitting as per Nomura et al. ${ }^{14)}$

For the clinical assessment, back pain and leg pain were assessed by the 10-point visual analog scale (VAS), Oswestry disability index (ODI), and walking distance without resting both before surgery and at 24-36 months after surgery. Intraoperative blood loss, operation time, and surgical complications were also investigated. All clinical parameters were collected retrospectively from previously assessed electronic medical records.

Data were analyzed using the SPSS ver. 18.0 (SPSS Inc., Chicago, IL, USA). A paired $t$-test was used to analyze the difference between the preoperative mean scores or values and the postoperative ones. The Mann-Whitney $U$-test was used to compare nonparametric variables and Pearson chi-square test was used to compare frequencies in the subgroups. The level of statistical significance was defined as a $p$-value $<0.05$ for a two-sided hypothesis. Data are presented as mean and standard deviation. 


\section{Surgical Procedure}

The authors' technique for posterior decompression of a lumbar spinal stenosis was performed with the following modification based on the spinous process-splitting laminectomy introduced by Watanabe et al..$^{10)}$ and modified by Cho et al., ${ }^{12)}$ Nomura et al., ${ }^{14)}$ and Hatta et al. ${ }^{23)}$

As an example of single-level decompression at L45 , in the prone position, a posterior midline skin incision was made starting from the tip of the L4 spinous process and extending 3-4 cm distally to expose the supraspinous ligament between the L4 and 5 spinous processes. After the sharp division of the supraspinous and interspinous ligaments with a scalpel, the total or distal half of the L4 spinous process was split longitudinally and evenly using an ultrasonic blade (BoneScalpel; Misonix Inc., Farmingdale, NY, USA) (Fig. 1A). The split part of the spinous process was then detached from the base of the L4 lamina and opened together with the sharply divided supraspinous and interspinous ligaments to expose the distal half of the L4 lamina, leaving the bilateral paraspinal muscles attached to the lateral aspect of the split spinous process and maintaining cortical, or at least periosteal, continuity between the remaining non-split and split parts of each L4 spinous process similar to a greenstick fracture. Retracting the split halves of the spinous process bilaterally, together with the attached paraspinal muscle, allowed us to obtain ample working space for decompression. Decompression was achieved in a partial laminectomy (or bilateral laminotomy) pattern, with partial removal of the distal part of the L4 lamina, the proximal part of the L5 lamina, and undercutting of the bony and ligamentous roof of the bilateral lateral recess until the thecal sac and traversing roots were thoroughly decompressed (Fig. 1B). Following decompression of the affected nerve roots and the thecal sac, meticulous haemostasis was performed before a drain was inserted. Split L4 spinous processes were reapproximated and sutured together with the split periosteum of the spinous process and the supraspinous and interspinous ligaments, and the wound was then closed layer by layer. For multilevel decompression, the most proximal spinous process was partially split in only the distal half and the next spinous processes were split totally as per Watanabe et al. ${ }^{10)}$ For example, to decompress L2-3-4-5, the distal half of the L2 spinous process and the total of the L3 and L4 spinous processes were split before a partial laminectomy of each level was performed (Fig. 1B).

\section{Postoperative Care}

Patients were allowed to sit up and walk without a brace or any other lumbar support 1 day after the operation. Patients were discharged after undergoing observation for a day following removal of the drain.

\section{Assessment of Bony Union}

The rate and pattern of bony union both between the split spinous processes and at the spinolaminar junction were analyzed using 2-mm slice CT images acquired at 6-18 months after surgery. The observation of bony continuity with no gap between split or detached bones on at least two slices of the index region was considered to represent union, and discontinuity observed in all sections was considered as nonunion. The union pattern was classified into three categories as follows: (1) complete union (complete restoration of the spinolaminar structure): union observed
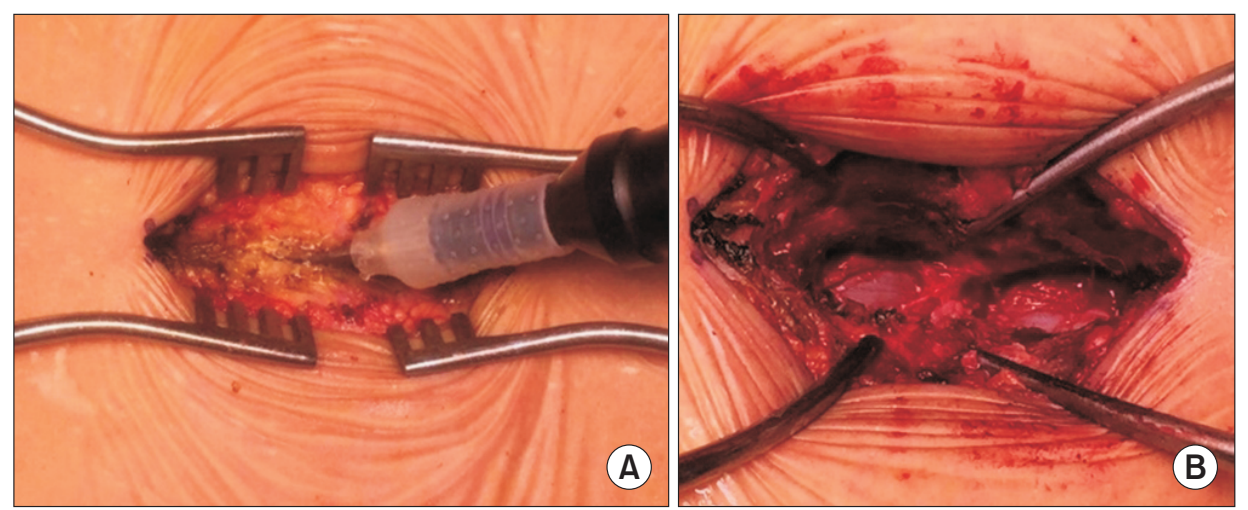

Fig. 1. Intraoperative photographs. (A) The spinous process was divided longitudinally and evenly using an ultrasonic blade. (B) The $L 4$ spinous process was split longitudinally in the midline and then divided at its base from the $L 4$ posterior arch, leaving the bilateral paraspinal muscles attached to the split spinous process. The supra- and interspinous ligaments between $L 3-4$ and $L 4-5$ were also split longitudinally using a scalpel. The split halves of the spinous process were bilaterally retracted with the Gelpi retractor to obtain ample working space in the L3-4 and L4-5 interlaminar spaces. The affected nerve tissues were successfully decompressed. 
both between the split spinous processes and at the spinolaminar junction; (2) partial union (floated union of the spinous process): union observed only between the split spinous processes and nonunion at the spinolaminar junction; (3) nonunion: no union observed either between the split spinous processes or at the spinolaminar junction.

\section{RESULTS}

A total of 73 patients who underwent posterior lumbar decompression via the split spinous process approach were included in the final analysis. The mean age at the time of operation was $67.7 \pm 9.5$ years. Thirty-nine patients underwent surgery for single-level disease, 24 for two levels, nine for three levels, and one for four levels (total number of levels, 118). The mean postoperative hospital stay was 3 \pm 1.2 days. The mean operation time per patient was 79.7 \pm 31.2 minutes, and the mean operation time per level was $53.3 \pm 19.4$ minutes. The mean estimated blood loss per patient was $145.6 \pm 105.3 \mathrm{~mL}$, and the estimated blood loss per level was $92.0 \pm 56.9 \mathrm{~mL}$. The mean follow-up period was $33.4 \pm 6.1$ months (Table 1 ).

No major morbidity or mortality was observed during the perioperative period and there were no cases of postoperative haematoma or infection. One patient experienced wound dehiscence and slight serosanguineous discharge without evidence of infection at 1 week after surgery; the wound was successfully reclosed. Three cases of pinpoint dural tear were noted during surgery and these were managed with a dural sealant without any subsequent complications. The preoperative mean VAS score for back pain was significantly decreased at 24-36 months after surgery from $4.5 \pm 2.1$ to $2.8 \pm 1.2(p<0.001)$. The VAS score for leg pain was also significantly decreased at 24-36 months after surgery, from 8.0. \pm 0.7 to $2.8 \pm 1.3$ ( $p$ $<0.001)$. The ODI score and walking distance were also significantly improved after surgery (ODI score: from 39.0 \pm 7.6 to $19.6 \pm 6.7, p<0.001$; walking distance: from 199.6 $\pm 231.8 \mathrm{~m}$ to $885.6 \pm 584.3 \mathrm{~m}, p<0.001$ ). The postoperative walking distance, in particular, was four times better than the preoperative walking distance (Fig. 2). Of a total of 118 levels of the split spinous processes, complete union was observed in 61 levels (51.7\%), partial union in $51 \mathrm{lev-}$ els (43.2\%), and nonunion in 6 levels (5.1\%) (Fig. 3).

Among the subgroups categorized according to the number of levels decompressed, the rates of union were as follows: for single-level decompression, the complete union rate was $71.8 \%$, the partial union rate was $25.6 \%$, and the nonunion rate was $2.6 \%$; for two-level decompression, the complete union rate was $47.9 \%$, the partial union rate was $47.9 \%$, and the nonunion rate was $4.2 \%$; and for three-level decompression, the complete union rate was $37.0 \%$, the partial union rate was $51.9 \%$, and the nonunion rate was $11.1 \%$. Among the subgroups categorized according to the extent of the split spinous process, the rates of union were as follows: for the partially split spinous process group, the complete union rate was $85.7 \%$, the partial union rate was $14.3 \%$, and the nonunion rate was $0 \%$; for the totally split spinous process group, the complete union rate was $32.9 \%$, the partial union rate was $59.2 \%$, and the nonunion rate was $7.9 \%$ (Table 2). None of the cases showed postoperative segmental instability of the lumbar spine at the 24-36 months of follow-up on simple radiography.

Table 1. Baseline Characteristics of the Patients

\begin{tabular}{|c|c|}
\hline Characteristic & Value \\
\hline Age at time of operation (yr) & $67.7 \pm 9.5(47-92)$ \\
\hline Male:female & $49: 24$ \\
\hline Body mass index $\left(\mathrm{kg} / \mathrm{m}^{2}\right)$ & $25.1 \pm 3.5(17.4-34.6)$ \\
\hline \multicolumn{2}{|l|}{ Level of operation } \\
\hline 1 & 39 \\
\hline 2 & 24 \\
\hline 3 & 9 \\
\hline 4 & 1 \\
\hline Total levels of operation & 118 \\
\hline Mean postoperative hospital day & $3 \pm 1.2(2-8)$ \\
\hline \multicolumn{2}{|l|}{ Operative time (min) } \\
\hline Per person & $79.7 \pm 31.2(30-170)$ \\
\hline Per level & $53.3 \pm 19.4(26-150)$ \\
\hline \multicolumn{2}{|l|}{ Estimated blood loss (mL) } \\
\hline Per person & $145.6 \pm 105.3(30-500)$ \\
\hline Per level & $92.0 \pm 56.9(25-400)$ \\
\hline Time to first ambulation after surgery (day) & $1.26 \pm 0.4(0-3)$ \\
\hline Follow-up period (mo) & $33.4 \pm 6.1(24-42)$ \\
\hline Postoperative CT scan (mo) & 73 \\
\hline $6-11$ & 53 \\
\hline $12-17$ & 11 \\
\hline 18 & 9 \\
\hline
\end{tabular}

Values are presented as mean \pm standard deviation (range). CT: computed tomography. 
Wi et al. Spinous Process-Splitting Laminectomy

Clinics in Orthopedic Surgery • Vol. 11, No. 1, 2019• www.ecios.org

A

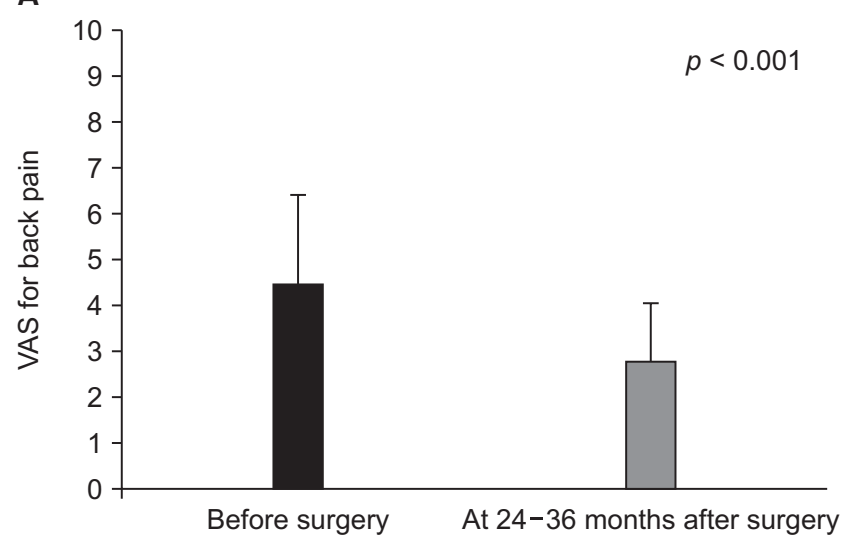

C

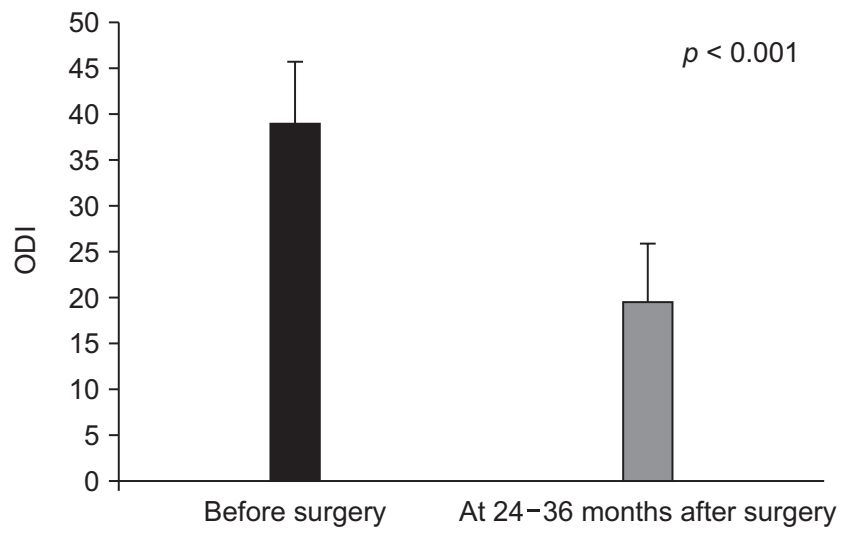

B

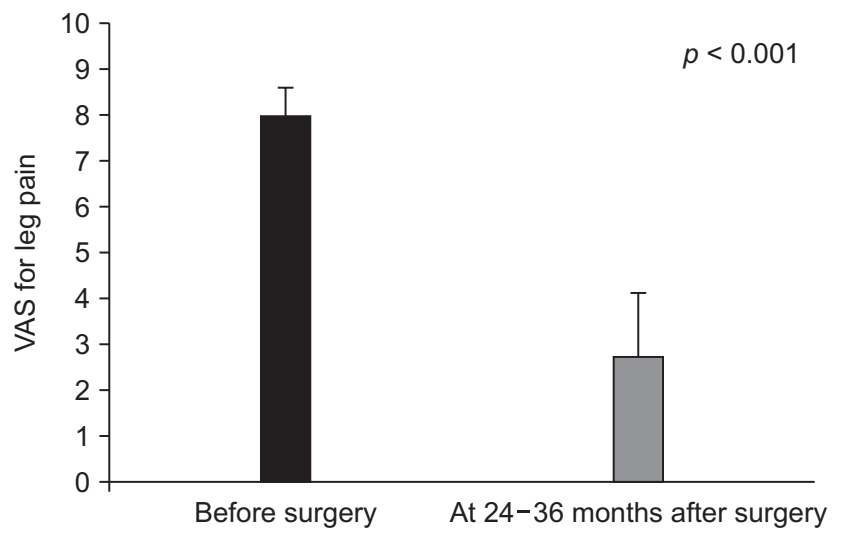

D

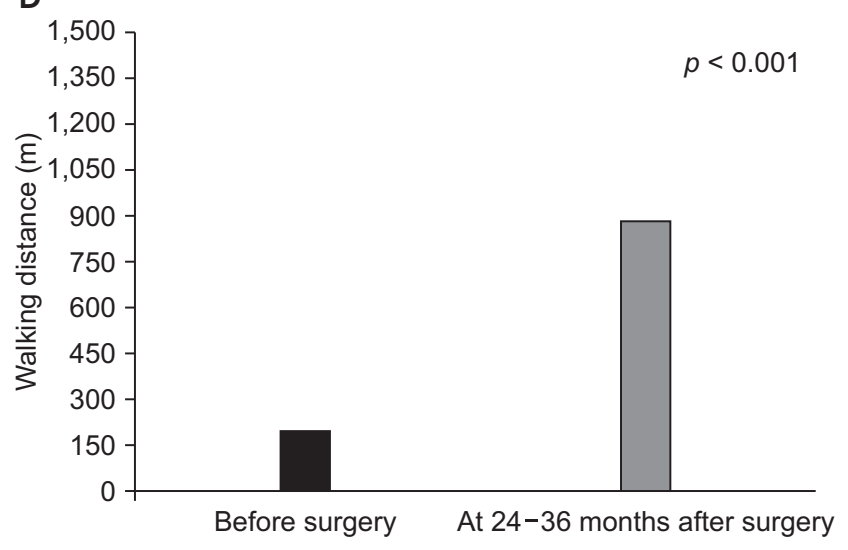

Fig. 2. Clinical outcomes. (A) Visual analog scale (VAS) for back pain. (B) VAS for leg pain. (C) Oswestry disability index (ODI). (D) Walking distance.
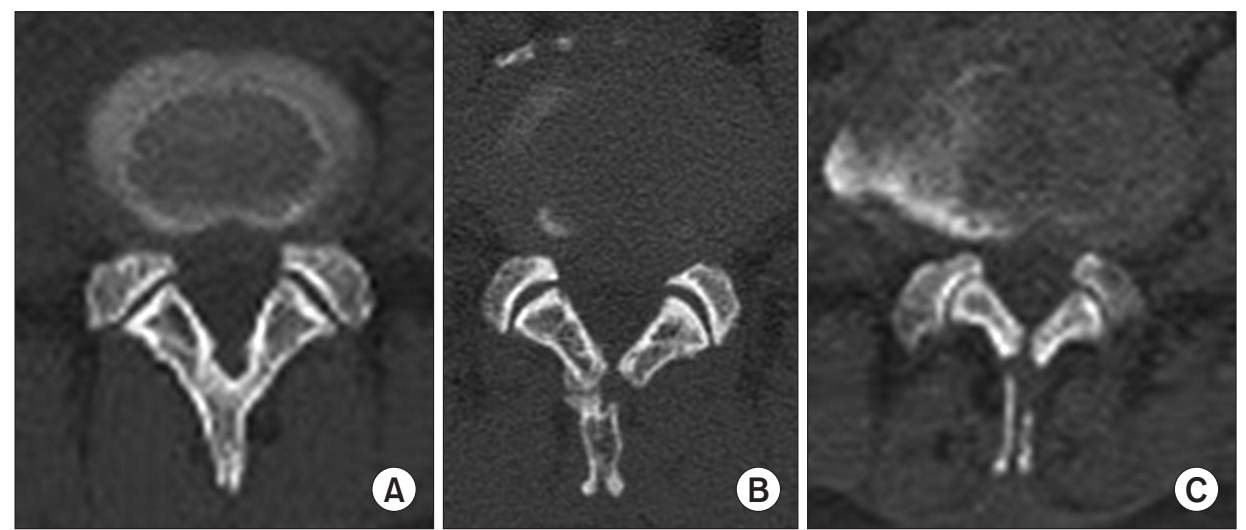

Fig. 3. Union pattern. (A) Complete union (complete restoration of spinolaminar structure): union observed both in-between split spinous processes and at the spinolaminar junction. (B) Partial union (floated union of spinous process): union observed only in-between split spinous processes and nonunion at the spinolaminar junction. (C) Nonunion: no union observed in-between split spinous processes and at the spinolaminar junction.

\section{DISCUSSION}

In the current study, the patients reported a markedly reduced disability index score and improved walking distance at 24-36 months of after surgery. Decompression of the lateral recess could be performed effectively with the surgeon standing on one side while undercutting and removing the hypertrophic facets and ligamentum flavum on the opposite side by tilting the operating table, while the use of a surgical microscope can also aid visualisation and illumination (Fig. 4). A satisfactory amount of decompression using a similar method has been reported to be achievable by other authors after analysing postoperative MRI. ${ }^{12,17)}$ 
Wi et al. Spinous Process-Splitting Laminectomy

Clinics in Orthopedic Surgery • Vol. 11, No. 1, 2019•www.ecios.org

Table 2. Union Rate and Pattern in Each Group

\begin{tabular}{|c|c|c|c|c|}
\hline Variable & Complete union & Partial union & Nonunion & Sum \\
\hline \multicolumn{5}{|l|}{ Group $1^{*}$} \\
\hline \multicolumn{5}{|c|}{ No. of decompressed level } \\
\hline 1 & 28 (71.8) & $10(25.6)$ & $1(2.6)$ & 39 \\
\hline 2 & $23(47.9)$ & $23(47.9)$ & $2(4.2)$ & 48 \\
\hline 3 & $10(37.0)$ & $14(51.9)$ & $3(11.1)$ & 27 \\
\hline 4 & 0 & $4(100)$ & 0 & 4 \\
\hline Total & $61(51.7)$ & $51(43.2)$ & $6(5.1)$ & 118 \\
\hline \multicolumn{5}{|l|}{ Group $2^{\dagger}$} \\
\hline \multicolumn{5}{|c|}{ Extent of spinous process splitting } \\
\hline Partial splitting & 36 (85.7) & $6(14.3)$ & 0 & 42 \\
\hline Total splitting & 25 (32.9) & $45(59.2)$ & $6(7.9)$ & 76 \\
\hline Total & $61(51.7)$ & $51(43.2)$ & $6(5.1)$ & 118 \\
\hline
\end{tabular}

Values are presented as number (\%).

${ }^{*}$ Statistically significant association was noted between the number of levels decompressed and union pattern (complete union vs. partial union): value, 9.999; $\mathrm{df}=2, p$-value $=0.007$ on Pearson chi-square test. ${ }^{\dagger}$ Statistically significant association was noted between the extent of splitting spinous process and union pattern (complete union vs. partial union): value, 26.461; $\mathrm{df}=1, p$-value $<0.001$ on Pearson chi-square test.
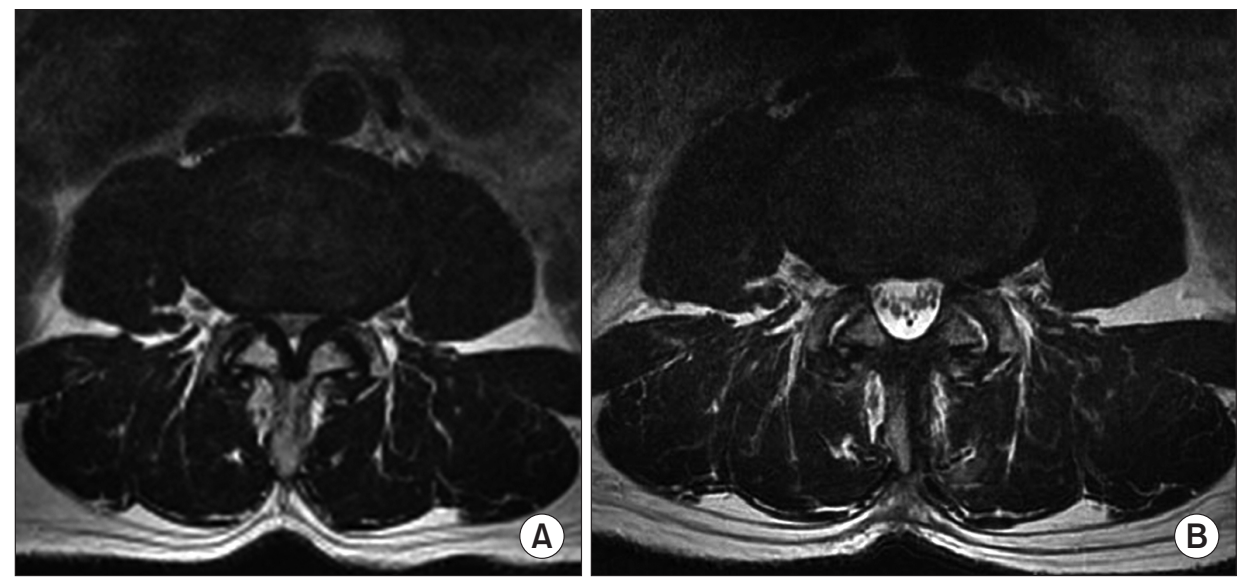

Fig. 4. T2-weighted axial magnetic resonance imaging. (A) The preoperative image revealed severe central and lateral recess stenosis. (B) After decompression, the spinous process and paraspinal muscles were seen well preserved at the L4-5 level.

In the current consecutive series of 73 cases, all complications including three incidental durotomies and one wound dehiscence occurred in the first 33 cases, and there were none in the final 40 cases. The reported complication rates following spinous process-splitting decompression by other authors are no higher than those of conventional decompression, where the complication rates range from $5 \%$ to $15 \%$. ${ }^{9,25)}$

Union between the split spinous process was observed in almost all cases (94.9\%) and complete restoration of the spinolaminar structure to maintain its lever-arm function as an attachment site of the paravertebral muscles was observed in up to $71.8 \%$ of single-level surgery cases and $51.7 \%$ of the overall levels in the current study. However, there were no significant differences in the clinical results (paired $t$-test for postoperative back pain $(p=1.000)$, ODI ( $p=0.223)$ and walking distance $(p=0.308)$, MannWhitney $U$-test for postoperative leg pain $(p=0.545)$ between patients who obtained complete restoration of the spinolaminar structure at all affected levels and those who obtained a nonunion only or floated spinous process. The trend of a higher rate of complete restoration of the spino- 
laminar structure was observed in single-level decompression cases and in the partially split spinous process group compared to multilevel decompression and in the totally split spinous process group. The rate of complete restoration of the spinolaminar structure was significantly high if the spinous process was split partially following Nomura et al.s technique, ${ }^{14)}$ rather than totally following the original technique by Watanabe et al. ${ }^{10)}(85.7 \%$ vs. $32.9 \%)$.

Nomura et al. ${ }^{14)}$ originally reported a union rate of up to $97.1 \%$ between the split spinous process and $82.7 \%$ between the spinous base and lamina. They also reported that the floated spinous process, nonunion at the spinolaminar junction, was observed more commonly in cases with spondylolisthesis but that this did not affect the clinical outcome in terms of the Japanese Orthopedic Association score. Considering the relatively high rate of union in Nomura et al's report, and as shown in the results of the comparison according to the extent of splitting in the current study, modification of the technique with partial splitting of the spinous process to maintain cortical or periosteal continuity between the split and non-split parts of the spinous process, attached to the remaining spinolaminar structure, is thought to provide a more favourable environment for bony union in terms of stability and a reduction in the gap to be healed. The ultrasonic scalpel is also thought to have provided several advantages for splitting the spinous process since the device is known to allow narrower bone cuts with minimal bone loss and soft tissue injury and reduced thermal injury, bleeding, and operating time. ${ }^{26,27)}$

The limitation of this study was that we were unable to establish the long-term effect of the preserved or non-preserved spinolaminar structure and paravertebral muscle attachment, such as segmental instability, recurrent stenosis, and related clinical outcomes due to the relatively short follow-up period. Kakiuchi and Fukushima, ${ }^{20)}$ in their retrospective study following spinous process-split open-door lumbar laminoplasty patients with a follow-up of more than 10 years, reported that although nonunion of the spinous process did not affect the mid-term clinical results at 2-4 years after surgery, it could have a deleterious effect on the long-term clinical outcomes, such as back and leg pain. Complete restoration of spinolaminar structure, as confirmed, can be achieved with a much higher likelihood when the spinous process is split partially, preserving part of spinolaminar junction during decompression with partial laminectomy, rather than total splitting of the spinous process.

This study provides evidence of the short-term benefits of the spinous process-splitting approach and addresses some of the factors associated with union of the split spinous process. We found that the single-level operation and partial splitting of the spinous process were favourable factors for obtaining complete restoration of the posterior bony structure of the lumbar spine in the spinous process-splitting decompression procedure. Although the short-term clinical outcomes of this procedure were positive, long-term follow-up and a prospective study of this subject should be conducted in the future.

\section{CONFLICT OF INTEREST}

No potential conflict of interest relevant to this article was reported.

\section{REFERENCES}

1. Verbiest H. A radicular syndrome from developmental narrowing of the lumbar vertebral canal. J Bone Joint Surg Br. 1954;36(2):230-7.

2. Kawaguchi Y, Matsui H, Gejo R, Tsuji H. Preventive measures of back muscle injury after posterior lumbar spine surgery in rats. Spine (Phila Pa 1976). 1998;23(21):2282-7.

3. Kawaguchi Y, Matsui H, Tsuji H. Back muscle injury after posterior lumbar spine surgery. Part 1: histologic and histochemical analyses in rats. Spine (Phila Pa 1976). 1994;19(22):2590-7.

4. Kawaguchi Y, Yabuki S, Styf J, et al. Back muscle injury after posterior lumbar spine surgery: topographic evaluation of intramuscular pressure and blood flow in the porcine back muscle during surgery. Spine (Phila Pa 1976).
1996;21(22):2683-8.

5. See DH, Kraft GH. Electromyography in paraspinal muscles following surgery for root compression. Arch Phys Med Rehabil. 1975;56(2):80-3.

6. Sihvonen T, Herno A, Paljarvi L, Airaksinen O, Partanen J, Tapaninaho A. Local denervation atrophy of paraspinal muscles in postoperative failed back syndrome. Spine (Phila Pa 1976). 1993;18(5):575-81.

7. Mariconda M, Zanforlino G, Celestino GA, Brancaleone S, Fava R, Milano C. Factors influencing the outcome of degenerative lumbar spinal stenosis. J Spinal Disord. 2000;13(2):131-7.

8. Radu AS, Menkes CJ. Update on lumbar spinal stenosis: ret- 
Wi et al. Spinous Process-Splitting Laminectomy

Clinics in Orthopedic Surgery • Vol. 11, No. 1, 2019• www.ecios.org

rospective study of 62 patients and review of the literature. Rev Rhum Engl Ed. 1998;65(5):337-45.

9. Silvers HR, Lewis PJ, Asch HL. Decompressive lumbar laminectomy for spinal stenosis. J Neurosurg. 1993;78(5):695701 .

10. Watanabe K, Hosoya T, Shiraishi T, Matsumoto M, Chiba K, Toyama Y. Lumbar spinous process-splitting laminectomy for lumbar canal stenosis: technical note. J Neurosurg Spine. 2005;3(5):405-8.

11. Watanabe K, Matsumoto M, Ikegami T, et al. Reduced postoperative wound pain after lumbar spinous process-splitting laminectomy for lumbar canal stenosis: a randomized controlled study. J Neurosurg Spine. 2011;14(1):51-8.

12. Cho DY, Lin HL, Lee WY, Lee HC. Split-spinous process laminotomy and discectomy for degenerative lumbar spinal stenosis: a preliminary report. J Neurosurg Spine. 2007;6(3):229-39.

13. Kim K, Isu T, Sugawara A, Matsumoto R, Isobe M. Comparison of the effect of 3 different approaches to the lumbar spinal canal on postoperative paraspinal muscle damage. Surg Neurol. 2008;69(2):109-13.

14. Nomura H, Yanagisawa Y, Arima J, Oga M. Clinical outcome of microscopic lumbar spinous process-splitting laminectomy: clinical article. J Neurosurg Spine. 2014;21(2):18794.

15. Uehara M, Takahashi J, Hashidate H, et al. Comparison of spinous process-splitting laminectomy versus conventional laminectomy for lumbar spinal stenosis. Asian Spine J. 2014;8(6):768-76.

16. Kanbara S, Yukawa Y, Ito K, Machino M, Kato F. Surgical outcomes of modified lumbar spinous process-splitting laminectomy for lumbar spinal stenosis. J Neurosurg Spine. 2015;22(4):353-7.

17. Lee S, Srikantha U. Spinous process splitting laminectomy: clinical outcome and radiological analysis of extent of decompression. Int J Spine Surg. 2015;9:20.

18. Baghdadi YM, Moussallem CD, Shuaib MA, Clarke MJ, Dekutoski MB, Nassr AN. Lumbar spinous process-splitting laminoplasty: a novel technique for minimally invasive lumbar decompression. Orthopedics. 2016;39(5):e950-6.

19. Maruo K, Tachibana T, Inoue S, Arizumi F, Yoshiya S. Prognostic factors of surgical outcome after spinous processsplitting laminectomy for lumbar spinal stenosis. Asian Spine J. 2015;9(5):705-12.

20. Kakiuchi M, Fukushima W. Impact of spinous process integrity on ten to twelve-year outcomes after posterior decompression for lumbar spinal stenosis: study of open-door laminoplasty using a spinous process-splitting approach. J Bone Joint Surg Am. 2015;97(20):1667-77.

21. Lee DY, Lee SH. Spinous process splitting laminectomy for lumbar canal stenosis: a critical appraisal. Minim Invasive Neurosurg. 2008;51(4):204-7.

22. Rajasekaran S, Thomas A, Kanna RM, Prasad Shetty A. Lumbar spinous process splitting decompression provides equivalent outcomes to conventional midline decompression in degenerative lumbar canal stenosis: a prospective, randomized controlled study of 51 patients. Spine (Phila $\mathrm{Pa}$ 1976). 2013;38(20):1737-43.

23. Hatta Y, Shiraishi T, Sakamoto A, et al. Muscle-preserving interlaminar decompression for the lumbar spine: a minimally invasive new procedure for lumbar spinal canal stenosis. Spine (Phila Pa 1976). 2009;34(8):E276-80.

24. Cammisa FP Jr, Girardi FP, Sangani PK, Parvataneni HK, Cadag S, Sandhu HS. Incidental durotomy in spine surgery. Spine (Phila Pa 1976). 2000;25(20):2663-7.

25. Wang JC, Bohlman HH, Riew KD. Dural tears secondary to operations on the lumbar spine: management and results after a two-year-minimum follow-up of eighty-eight patients. J Bone Joint Surg Am. 1998;80(12):1728-32.

26. Hu X, Ohnmeiss DD, Lieberman IH. Use of an ultrasonic osteotome device in spine surgery: experience from the first 128 patients. Eur Spine J. 2013;22(12):2845-9.

27. Sanborn MR, Balzer J, Gerszten PC, Karausky P, Cheng BC, Welch WC. Safety and efficacy of a novel ultrasonic osteotome device in an ovine model. J Clin Neurosci. 2011;18(11):1528-33. 